\title{
Clinical Vignettes \\ Hormones and the Bone Marrow: Panhypopituitarism and Pancytopenia in a Man with a Pituitary Adenoma
}

\author{
Dianna Lang, B.Sc., Jennifer S. Mead, P.A-.C., and David B. Sykes, M.D., Ph.D. \\ Division of Hematology, Massachusetts General Hospital Cancer Center, Boston, MA, USA.
}

In rare cases, pancytopenia results from hormonal deficiencies that arise in the setting of panhypopituitarism. Here we describe the unusual case of a 60-year-old man who presented with progressive fatigue and polyuria, and whose laboratory workup revealed a deficiency of the five hormones associated with the action of the anterior pituitary (thyroid hormone, testosterone, cortisol, prolactin, and insulin-like growth factor-1). Imaging of the pituitary demonstrated a cystic mass consistent with a pituitary adenoma replacing much of the normal pituitary tissue. His symptoms and hematologic abnormalities rapidly resolved with prednisone and levothyroxine supplementation. While the majority of reported cases of panhypopituitarism with bone marrow suppression are the result of peripartum sepsis or hemorrhage leading to pituitary gland necrosis (Sheehan's syndrome), it is also important to consider the diagnosis of hypopituitarism in patients with hypothyroidism, low cortisol levels, and pancytopenia. The causal relationship between pancytopenia and panhypopituitarism is not well understood, though it does reinforce the important influence of these endocrine hormones on the health of the bone marrow.

KEY WORDS: pancytopenia; panhypopituitarism; hypopituitarism; Sheehan's syndrome; pituitary adenoma.

J Gen Intern Med 30(5):692-6

DOI: $10.1007 / \mathrm{s} 11606-014-3161-\mathrm{x}$

(c) Society of General Internal Medicine 2015

\section{CASE PRESENTATION}

A 60-year-old man was admitted to Massachusetts General Hospital with chest pain thought to be the result of his longstanding gastroesophageal reflux disease. He had a history of glaucoma, atypical nevi, and diverticulosis. He reported progressively worsening fatigue and weakness, a $10 \mathrm{lb}$ weight loss, polyuria, and increased thirst. Laboratory testing on presentation was notable for hyponatremia (116 mmol/L) and pancytopenia. Out of concern for an underlying malignancy, the patient underwent contrast-enhanced computed tomography scans of the chest, abdomen, and pelvis, which were unremarkable. Given his progressive fatigue and hyponatremia, a morning serum cortisol level was checked and found to be very depressed $(1.0 \mu \mathrm{g} / \mathrm{dL}$; normal range 5-25 $\mu \mathrm{g} / \mathrm{dL})$. This prompted a more extensive endocrine evaluation (Table 1) that resulted in documentation of low levels of thyroid hormone, cortisol, testosterone, prolactin, and

Published online January 13, 2015 insulin-like growth factor-1 (IGF-1). A diagnosis of panhypopituitarism was made based on the reduced levels of all five hormones associated with the action of the anterior pituitary. A cosyntropin stimulation test demonstrated an inappropriately low increase in serum cortisol levels (1.2 to $7.4 \mu \mathrm{g} / \mathrm{dL}$ ), suggesting reduced adrenal responsiveness due to prolonged adrenocorticotropic hormone (ACTH) deficiency. Though euvolemic, the patient displayed hyponatremia, with elevated urine osmolality, suggesting a component of elevated antidiuretic hormone (ADH) thought to be compensatory in the setting of glucocorticoid deficiency.

In evaluation of the pituitary gland, MRI demonstrated a $6 \mathrm{~mm}$ cystic lesion in the posterior aspect of the pituitary (Fig. 1a). The radiologic appearance was most consistent with a cystic pituitary adenoma compressing and replacing the normal tissue of the pituitary gland. In addition, a small enhancing tectal mass was incidentally noted that was believed to represent a tectal glioma (Fig. 1b). A lumbar puncture revealed normal cerebrospinal fluid protein and glucose levels, and one nucleated cell (normal range $0-5 / \mathrm{mm}^{3}$ ). CSF cytology test results revealed no malignant cells. Results of repeat MRI exams performed 4 and 8 months later demonstrated stability of both the pituitary adenoma and tectal glioma.

The patient's complete blood count and peripheral blood smear demonstrated normocytic and normochromic anemia. There were no schistocytes, spherocytes, teardrop red blood cells, or nucleated red blood cells. The white blood cells showed a normal differential, with no immature forms. The platelet count was reduced $\left(125,000 / \mathrm{mm}^{3}\right)$ compared to baseline $\left(210,000 / \mathrm{mm}^{3}\right)$. Treatment with prednisone $(4 \mathrm{mg} /$ day $)$ and levothyroxine (125 $\mathrm{\mu g} /$ day), administered orally, was initiated. The patient's symptoms of fatigue, as well as his hematologic abnormalities, resolved rapidly (Table 1).

\section{DISCUSSION}

The relationship between pancytopenia and the hormones of the anterior pituitary is poorly understood. Panhypopituitarism refers to a deficiency of the five hormones produced in the anterior pituitary and released from the hypothalamus: somatotropin (growth hormone $[\mathrm{GH}]$ ), thyrotropin (thyroidstimulating hormone $[\mathrm{TSH}]$ ), corticotropin (adrenocorticotropic hormone $[\mathrm{ACTH}]$ ), lactotropin (prolactin [PRL]), and gonadotropin (luteinizing hormone [LH] and folliclestimulating hormone $[\mathrm{FSH}]$ ). A direct and causal relationship 
Table 1 Laboratory Values for Described 60-Year-Old Male Patient at Baseline, 7 Months Prior to Hospital Admission, upon Admission, at the Time of Discharge, and in Outpatient Follow-Up Visits

\begin{tabular}{|c|c|c|c|c|c|c|c|c|}
\hline & Baseline & & Admission & Discharge & Follow-up & Follow-up & Follow-up & \\
\hline Result & $10 / 12 / 10$ & $05 / 08 / 13$ & $01 / 20 / 14$ & $01 / 24 / 14$ & $03 / 04 / 14$ & $05 / 13 / 14$ & 11/18/14 & $\begin{array}{l}\text { Normal } \\
\text { Range }\end{array}$ \\
\hline \multicolumn{9}{|l|}{ Complete blood count } \\
\hline WBC $\left(1000 / \mathrm{mm}^{3}\right)$ & 6 & 5.1 & 3.3 & 7.7 & 7.6 & 6.7 & 7.3 & $4.5-11.0$ \\
\hline Hemoglobin (g/dL) & 14.5 & 13 & 13 & 13.3 & 12.1 & 13.4 & 13.9 & $13.5-17.5$ \\
\hline Hematocrit $(\%)$ & 41.8 & 36.6 & 35.1 & 36.5 & 37.8 & 39.6 & 42.8 & $41.0-53.0$ \\
\hline MCV (fL) & 89 & 87 & 83 & 84 & 95 & 90 & 95 & 80-100 \\
\hline Platelet $\left(1000 / \mathrm{mm}^{3}\right)$ & 210 & 182 & 125 & 166 & 197 & 187 & 200 & $150-450$ \\
\hline Reticulocyte (\%) & & & 1.5 & & & & 1.4 & $0.5-2.5$ \\
\hline Reticulocyte index * & & & 1.2 & & & & 1.5 & \\
\hline Reticulocyte production index ** & & & 0.8 & & & & 1.5 & \\
\hline $\operatorname{ESR}(\mathrm{mm} / \mathrm{h})$ & & & 13 & & 2 & & & $0-13$ \\
\hline Iron $(\mu \mathrm{g} / \mathrm{dL})$ & 82 & 113 & 30 & & & 80 & 96 & $45-160$ \\
\hline TIBC $(\mu \mathrm{g} / \mathrm{dL})$ & 289 & 222 & 205 & & & 223 & 237 & $230-404$ \\
\hline Ferritin $(\mu \mathrm{g} / \mathrm{L})$ & 491 & 724 & 1127 & & & 693 & 701 & $30-300$ \\
\hline \multicolumn{9}{|l|}{ Electrolytes } \\
\hline Sodium $(\mathrm{mmol} / \mathrm{L})$ & & & 116 & 133 & & & 141 & $135-145$ \\
\hline Potassium (mmol/L) & & & 3.9 & 3.6 & & & 4.1 & $3.4-4.8$ \\
\hline Chloride $(\mathrm{mmol} / \mathrm{L})$ & & & 84 & 96 & & & 99 & $100-108$ \\
\hline $\mathrm{CO}_{2}(\mathrm{mmol} / \mathrm{L})$ & & & 20 & 24 & & & 26 & $23.0-31.9$ \\
\hline $\begin{array}{l}\text { Blood urea nitrogen } \\
\text { (BUN) (mg/dL) }\end{array}$ & & & 9 & 11 & & & 16 & $8-25$ \\
\hline Creatinine $(\mathrm{mg} / \mathrm{dL})$ & & & 0.8 & 0.8 & & & 1.0 & $0.60-1.50$ \\
\hline Glucose $(\mathrm{mg} / \mathrm{dL})$ & & & 90 & 86 & & & 78 & $70-110$ \\
\hline \multicolumn{9}{|l|}{ Endocrine studies } \\
\hline Cortisol $(\mu \mathrm{g} / \mathrm{dL})$ & & & 1 & & & 2.7 & & $\begin{array}{c}5-25 \\
\text { (8 a.m.-noon) }\end{array}$ \\
\hline$(\mathrm{TSH})(\mu \mathrm{U} / \mathrm{mL})$ & & & 2.3 & & & 0.73 & 0.82 & $0.40-5.00$ \\
\hline Free thyroxine $\left(\mathrm{FT}_{4}\right)(\mathrm{ng} / \mathrm{dL})$ & & & 0.5 & & & 1.3 & & $0.91-1.8$ \\
\hline IGF-1 (somatomedin C) (ng/mL) & & & 17 & & & 51 & & $41-279$ \\
\hline Prolactin $(\mathrm{ng} / \mathrm{mL})$ & & & 1.8 & & & & & $0.00-15.0$ \\
\hline Testosterone (ng/dL) & & & 144 & & & 482 & 350 & $270-1070$ \\
\hline $\mathrm{ACTH}(\mathrm{pg} / \mathrm{mL})$ & & & 7 & & & & & $6-76$ \\
\hline Serum osm. (mOsm/kg) & & & 252 & & & & & 280-296 \\
\hline Urine sodium $(\mathrm{mmol} / \mathrm{L})$ & & & 200 & & & & & \\
\hline Urine osm. (mOsm/kg) & & & 752 & & & & & \\
\hline
\end{tabular}

* The reticulocyte index is calculated by multiplying the patient's reticulocyte count by the patient's hematocrit (Hct) divided by the expected normal hematocrit.

**The reticulocyte production index is calculated by dividing the reticulocyte index by a maturation factor to take into account the longer life span of prematurely released reticulocytes into the bloodstream. The maturation factor is equal to 1 (Hct 36-45\%), 1.5 (Hct 26-35\%), or 2 (Hct 16-25\%). The reticulocyte production index is considered low if it is less than $2 \%$ at a time when the patient is anemic.

ACTH adrenocorticotropic hormone, ESR erythrocyte sedimentation rate, MCV mean corpuscular volume, TIBC total iron-binding capacity, WBC white blood count

between bone marrow health and the pituitary seems likely, given the rapid resolution of hematologic abnormalities with hormone replacement therapy. ${ }^{1-10}$

In humans, isolated hormone deficiencies can affect hematopoiesis. Severe hypothyroidism can result in normocytic anemia. Men with hypogonadism (in particular, those who have undergone surgical orchiectomy) develop a mild and stable normocytic anemia ${ }^{11}$ where hemoglobin and hematocrit values generally fall into the normal female reference range, underscoring the role of androgens in erythropoiesis. Isolated deficiencies of the other hormones under the control of the anterior pituitary (cortisol, growth hormone, and prolactin) have not been reported to cause bone marrow abnormalities. However, growth hormone replacement treatment in patients with growth hormone deficiencies has been shown to increase levels of erythropoietin, though the mechanism through which this occurs is unknown. ${ }^{12}$ In vitro experiments have suggested that IGF-1 (under the control of growth hormone) leads to proliferation of immature erythroid colonies. ${ }^{13}$

The hematopoietic changes described in animal models of hypopituitarism do not faithfully represent the human experience, and significant species-specific differences likely exist. In one rat model, total hypophysectomy resulted in normocytic anemia that was completely reversed following treatment with cortisol and 

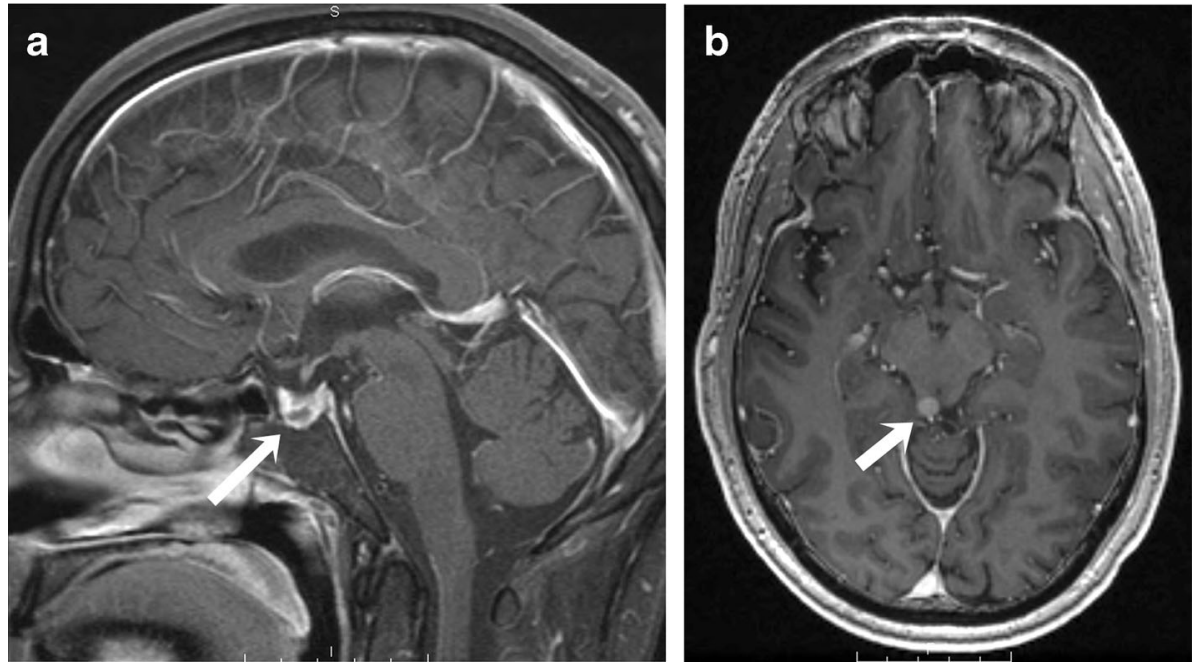

Fig. 1 Post-contrast MRI revealed (a) a cystic pituitary adenoma (sagittal image, white arrow), as well as (b) an incidental finding of a small enhancing tectal mass (axial image, white arrow) thought to represent a tectal glioma.

thyroid hormone replacement. ${ }^{14}$ The addition of supplemental growth hormone led to an increase in immature erythroid cells in the bone marrow, albeit no increase in the peripheral red blood cell count. Importantly, hypophysectomy in this study did not result in leukopenia or in a loss of bone marrow myeloid elements, in contrast to what is seen in human patients. In a second study of hypophysectomized rats, the rats developed anemia, leukopenia, and thrombocytopenia, all of which were fully reversible. ${ }^{15}$ In this model, the rats did not respond to thyroid hormone treatment, but did show a partial response to treatment with supplemental prolactin, a finding that is not consistent with studies in mice ${ }^{16}$ and humans. Of note, these studies used two different inbred strains of rats, which may have contributed to the very different findings.

In humans, pancytopenia as the result of hypopituitarism is a rare occurrence, and our review of the published literature (via PubMed and Google, using the keywords "anemia," "pancytopenia," "hypopituitarism," and "panhypopituitarism") revealed a total of only 21 published cases. Of these, the majority (15 of $21,{ }^{1-3,5-8,10,17-20,21-23}$ ) were reports of Sheehan's syndrome, a condition that occurs in women as a result of anterior pituitary hemorrhage and subsequent necrosis in the setting of hypotension due to blood loss during or after childbirth. In contrast, our patient was a 60 -year-old man whose panhypopituitarism was secondary to a pituitary adenoma. The end result, however, is thought to be the same: loss of the cells of the anterior pituitary due to the compressive effects of a pituitary mass or secondary to pituitary hemorrhage.

In addition to peripartum pituitary hemorrhage, panhypopituitarism can result from infection, stroke, head trauma, hypothalamic dysfunction, and primary or metastatic pituitary tumors. ${ }^{24}$ While these conditions may result in a deficiency of the anterior pituitary hormones, the hormones produced in the hypothalamus but secreted from the intermediate and posterior pituitary (antidiuretic hormone $[\mathrm{ADH}]$, oxytocin, and melanocyte-stimulating hormone [MSH]) are generally spared. The differential sensitivity of the anterior and posterior pituitary is likely the result of differences in their respective blood supplies - the anterior pituitary receives its blood supply from the superior hypophyseal artery, while the posterior pituitary receives a dual blood supply from both the superior and inferior hypophyseal arteries.

Many conditions can result in pancytopenia. Though our patient did not undergo a bone marrow biopsy evaluation, his normal mean corpuscular volume and lack of immature or dysplastic white blood cells in the peripheral blood smear argued against a myelodysplastic process. The absence of teardrop or nucleated red blood cells on evaluation of the peripheral smear, while not conclusive, tended to rule out an infiltrative bone marrow process. His vitamin B12 and folate levels were normal, and his low reticulocyte production index suggested that the anemia was secondary to decreased bone marrow production rather than to a destructive process. Of note, our patient had no new medication or toxin exposures and no evidence of an infectious process. Furthermore, he had a rapid bone marrow response to cortisol and thyroid hormone replacement therapy (Table 1), empirically confirming the link between his panhypopituitarism and pancytopenia. It is also interesting to note that his testosterone level normalized without supplementation (Table 1), suggesting an interconnection between hypothyroidism, low cortisol, and hypogonadism.

Much of what we know about pancytopenia stems from individual case reports or case series and from the empiric response of patients to hormone replacement treatment. As mentioned earlier, the majority of patients described have been women with Sheehan's syndrome. The history of Sheehan's syndrome was reviewed in a recent study, ${ }^{25}$ with the first case described in 1913. The condition has also been referred to as Simmonds' disease, named after Dr. Morris Simmonds, who described pituitary atrophy in a woman who died many years 
after the delivery of her fifth child, a delivery that was complicated by puerperal sepsis. $^{23}$ In 1937, Dr. Harold Leeming Sheehan conducted autopsies on women who had died of hemorrhagic shock in late pregnancy or shortly after delivery, and reported that destruction of the anterior pituitary was observed in 12 of 76 women. ${ }^{25}$

The first reported case of pancytopenia in the context of hypopituitarism was that of a 45-year-old woman who died in a comatose state 20 years after the birth of her daughter. Her autopsy revealed a hemorrhagic pituitary cyst and bone marrow aplasia. ${ }^{17}$ It is now recognized that the symptoms of Sheehan's syndrome may not manifest for many years following childbirth. A case series of 14 women found a mean duration of 18 years (range 1-33 years) before symptoms appeared, ${ }^{26}$ and another series of 65 cases of Sheehan's syndrome reported that $80 \%$ of the women presented with normochromic and normocytic anemia. ${ }^{22}$ Consistent with our case, hyponatremia is the most common electrolyte abnormality (33-69 \% of cases), and is believed to be multifactorial in nature (due to hypothyroidism, volume depletion, and cortisol deficiency). ${ }^{21}$

The treatment of pancytopenia and panhypopituitarism is also best studied in patients with Sheehan's syndrome. In 1975, a woman presenting 6 years after antepartum hemorrhage was successfully treated using a combination of cortisol, thyroid extract, estrogen, and progesterone, leading to normalization of blood counts. ${ }^{5}$ Another woman presenting 26 years after delivery received 6 weeks of supplemental treatment with levothyroxine and hydrocortisone, which reversed her bone marrow abnormalities. ${ }^{1}$ Three women (aged 22, 30, and 34 years) with pancytopenia that developed 2-8 years following delivery, $^{2}$ as well as a 55 -year-old woman with Sheehan's syndrome and isolated anemia, ${ }^{3}$ also demonstrated complete resolution of bone marrow abnormalities with glucocorticoid and thyroid hormone replacement therapy.

As mentioned previously, less common conditions can also result in damage to the anterior pituitary and subsequent pancytopenia, including reported cases of hypothalamic glioma, ${ }^{27}$ macroprolactinoma, ${ }^{4}$ empty sella syndrome, ${ }^{28}$ and suprasellar germinoma, ${ }^{9}$ as well as in patients following pituitary surgery and radiation for a functional macroadenoma $^{29}$ and where the condition was idiopathic. ${ }^{30}$ In a case report similar to our own, a 46-year-old man with pancytopenia in the setting of hypopituitarism caused by a macroprolactinoma had resolution of his bone marrow abnormalities with combination treatment that included hydrocortisone, levothyroxine, testosterone, and cabergoline. ${ }^{4}$

These cases illustrate the successful treatment of pancytopenia using a combination of glucocorticoid and thyroid hormone replacement. Little is known about the individual contributions of these hormones, and two cases in particular provide contrasting evidence of the bone marrow's response to treatment. A 40-year-old woman with Sheehan's syndrome presented with pancytopenia 12 years after delivery. She was started on glucocorticoid replacement therapy alone in the setting of normal TSH levels. In her case, complete resolution of pancytopenia occurred within 12 weeks, without the addition of supplemental thyroid hormone treatment. ${ }^{8}$ A 28-yearold man with acromegaly underwent excision of a pituitary macroadenoma, followed by radiation therapy; 6 years later, he presented with pancytopenia and hypopituitarism (with the exception of preserved thyrotropin). ${ }^{29}$ The patient was treated with hydrocortisone replacement therapy, and the pancytopenia resolved. However, in a somewhat different case, an 11year-old girl who developed pancytopenia following radiotherapy for a suprasellar germinoma ${ }^{9}$ was initially treated with glucocorticoid replacement and desmopressin, but pancytopenia persisted. The addition of levothyroxine - despite normal TSH levels - led to the rapid resolution of the pancytopenia, which argues for a critical role played by thyroid hormones in hematopoiesis. And as noted above, the utility of animal models may be questionable, given the very different responses in rats (after hypophysectomy) and humans to hormone supplementation. ${ }^{14,15}$

In conclusion, panhypopituitarism should be considered in women with unexplained pancytopenia who have a history of severe blood loss during childbirth, or in men or women who present with insidious lethargy, hyponatremia, and hypothyroidism. In these cases, an unrevealing workup for malignancy should prompt formal laboratory evaluation of all of the hormones of the anterior pituitary. The hematologic abnormalities would be expected to resolve rapidly with cortisol and thyroid hormone supplementation, though the differential importance of these remains in question. Should the pancytopenia not completely resolve, a bone marrow biopsy should be performed to evaluate for other causes.

Acknowledgments: We would like to thank Dr. Walter "Sunny" Dzik and Dr. Benjamin Drapkin for their guidance in preparing our manuscript and for their thoughtful care of the patient.

Conflicts of Interest: The authors each declare that they have no conflicts of interest.

Corresponding Author: David B. Sykes, M.D., Ph.D.; Division of Hematology Massachusetts General Hospital Cancer Center, Yawkey 7B, 55 Fruit Street, Boston, MA 02114, USA (e-mail: dbsykes@partners.org).

\section{REFERENCES}

1. Fatma M, Mouna E, Nabila R, Mouna M, Nadia C, Mohamed A. Sheehan's syndrome with pancytopenia: a case report and review of the literature. J Med Case Rep. 2011;5:490. doi:10.1186/1752-1947-5-490.

2. Laway BA, Bhat JR, Mir SA, Khan RS, Lone MI, Zargar AH. Sheehan's syndrome with pancytopenia-complete recovery after hormone replacement (case series with review). Ann Hematol. 2010;89(3):305-8. doi:10. 1007/s00277-009-0804-9.

3. Kim DY, Kim JH, Park YJ, Jung KH, Chung HS, Shin S, et al. Case of complete recovery of pancytopenia after treatment of hypopituitarism. Ann Hematol. 2004;83(5):309-12. doi:10.1007/s00277-003-0800-4.

4. Holmes GI, Shepherd P, Walker JD. Panhypopituitarism secondary to a macroprolactinoma manifesting with pancytopenia: case report and literature review. Endocr Pract Off J Am Coll Endocrinol Am Assoc Clin Endocrinologists. 2011;17(2):e32-6. doi:10.4158/EP10298.CR.

5. Ferrari E, Ascari E, Bossolo PA, Barosi G. Sheehan's syndrome with complete bone marrow aplasia: long-term results of substitution therapy with hormones. Br J Haematol. 1976;33(4):575-82. 
6. Gokmen Akoz A, Atmaca H, Ustundag Y, Ozdamar SO. An unusual case of pancytopenia associated with Sheehan's syndrome. Ann Hematol. 2007;86(4):307-8. doi:10.1007/s00277-006-0233-y.

7. Kim SK, Oh YJ, Joo PY, Kim YW, Hong SB, Kim MR, Nam MS, Kim YS. A case of pancytopenia caused by Sheehan's syndrome improved with hormone replacement therapy. J Korean Soc Endocrinol. 2000;15(45):595-9.

8. Laway BA, Mir SA, Bhat JR, Lone MI, Samoon J, Zargar AH. Hematological response of pancytopenia to glucocorticoids in patients with Sheehan's syndrome. Pituitary. 2012;15(2):184-7. doi:10.1007/s11102011-0304-5.

9. Lee AC. Pancytopenia secondary to hypopituitarism may just be due to hypothyroidism alone. Ann Hematol. 2010;89(11):1181. doi:10.1007/ s00277-010-0938-9.

10. Ozdogan M, Yazicioglu G, Karadogan I, Cevikol C, Karayalcin U, Undar L. Sheehan's syndrome associated with pancytopenia due to marrow aplasia; full recovery with hormone replacement therapy. Int $\mathrm{J}$ Clin Pract. 2004;58(5):533-5

11. Fonseca R, Rajkumar SV, White WL, Tefferi A, Hoagland HC. Anemia after orchiectomy. Am J Hematol. 1998;59(3):230-3.

12. Sohmiya M, Kato Y. Effect of long-term administration of recombinant human growth hormone (rhGH) on plasma erythropoietin (EPO) and haemoglobin levels in anaemic patients with adult GH deficiency. Clin Endocrinol. 2001;55(6):749-54.

13. Merchav S, Tatarsky I, Hochberg Z. Enhancement of erythropoiesis in vitro by human growth hormone is mediated by insulin-like growth factor I. Br J Haematol. 1988;70(3):267-71.

14. Meineke HA, Crafts RC. Effect of combined thyroxin-cortisone-growth hormone therapy on hematopoiesis in hypophysectomized rats. Proc Soc Exp Biol Med Soc Exp Biol Med. 1957;96(1):74-9.

15. Nagy E, Berczi I. Pituitary dependence of bone marrow function. $\mathrm{Br} \mathrm{J}$ Haematol. 1989;71(4):457-62.

16. Horseman ND, Zhao W, Montecino-Rodriguez E, Tanaka M, Nakashima $\mathbf{K}$, Engle SJ, et al. Defective mammopoiesis, but normal hematopoiesis, in mice with a targeted disruption of the prolactin gene. EMBO J. 1997;16(23):6926-35. doi:10.1093/emboj/16.23.6926.

17. Bloom A, Bryson CC. Aplastic anaemia in Simmonds's disease; report of a case. Br Med J. 1948;2(4566):75-7.

18. Dharmshaktu P, Bhowmick J, Manglani D, Dhanwal DK. Recurrent symptomatic hypoglycaemia with pancytopenia as a delayed presentation of Sheehan's syndrome with complete recovery after hormone replacement. BMJ Case Rep. 2013. doi:10.1136/bcr-2013-009014.

19. Atmaca H, Bayraktaroglu T, Gokmen Akoz A, Koyuncu BU. Two unusual cases with pancytopenia associated with Sheehan's syndrome. Endocr Abstr. 2008; 16:P151.

20. Pines Corrales PJ, Anton Bravo T, Zurita Sepulveda P. Pancytopenia and acquired factor IX deficiency in patient with Sheehan's syndrome. Med Clin. 2006; 127(11):439.

21. Anfuso S, Patrelli TS, Soncini E, Chiodera P, Fadda GM, Nardelli GB. A case report of Sheehan's syndrome with acute onset, hyponatremia and severe anemia. Acta Biomed Atenei Parmensis. 2009;80(1):736.

22. Gokalp D, Tuzcu A, Bahceci M, Arikan S, Bahceci S, Pasa S. Sheehan's syndrome as a rare cause of anaemia secondary to hypopituitarism. Ann Hematol. 2009;88(5):405-10. doi:10.1007/s00277-008-0607-4

23. Simmonds M. Über Hypophysisschwund mit tödlichem Ausgang. Dtsch Med Wochenschr. 1914;40(7):322-3.

24. van Aken MO, Lamberts SW. Diagnosis and treatment of hypopituitarism: an update. Pituitary. 2005;8(3-4):183-91. doi:10.1007/s11102-006-6039-z.

25. Tessnow AH, Wilson JD. The changing face of Sheehan's syndrome. Am J Med Sci. 2010;340(5):402-6. doi:10.1097/MAJ.0b013e3181f8c6df.

26. Huang YY, Ting MK, Hsu BR, Tsai JS. Demonstration of reserved anterior pituitary function among patients with amenorrhea after postpartum hemorrhage. Gynecol Endocrinol Off J Int Soc Gynecol Endocrinol. 2000; 14(2):99-104.

27. Badawi MA, Salih F, Al-Humaidi AA, El Khalifa MY, Elhadd TA. Bone marrow hypoplasia responsive to testosterone therapy in a patient with panhypopituitarism: need for adherence to androgen replacement. Endocr Pract Off J Am Coll Endocrinol Am Assoc Clin Endocrinologists. 2008;14(2):229-32. doi:10.4158/EP.14.2.229.

28. Foppiani L, Ruelle A, Guilici P, Del Monte P. Hypopituitarism in the elderly: two case-reports with heterogeneous presentation. Aging Clin Exp Res. 2009;21(1):76-81.

29. Koh JH, Lee YJ, Kang JH, Choi BK, Jeon YK, Kim SS, et al. Pancytopenia associated with hypopituitarism in an acromegaly patient: a case report and a review of the literature. Endocrinol Metab. 2012;27(4):308-13.

30. Killmann SA. A case of pancytopenia in hypopituitarism; with a review of the hematological changes in clinical and experimental pituitary insufficiency. Acta Haematol. 1956;15(1):34-45. 